\title{
Interleukin-7 is produced by afferent lymphatic vessels and supports lymphatic drainage
}

\author{
Maria Iolyeva, ${ }^{1}$ David Aebischer, ${ }^{1}$ Steven T. Proulx, ${ }^{1}$ Ann-Helen Willrodt, ${ }^{1}$ Tatiana Ecoiffier, ${ }^{2}$ Simone Häner, ${ }^{1}$ \\ Grégory Bouchaud, ${ }^{3}$ Carsten Krieg, ${ }^{3}$ Lucas Onder, ${ }^{4}$ Burkhard Ludewig, ${ }^{4}$ Laura Santambrogio,,${ }^{5}$ Onur Boyman, ${ }^{3}$ Lu Chen, ${ }^{2}$ \\ Daniela Finke, ${ }^{6}$ and Cornelia Halin ${ }^{1}$ \\ ${ }^{1}$ Institute of Pharmaceutical Sciences, Swiss Federal Institute of Technology, ETH Zurich, Zurich, Switzerland; ${ }^{2}$ Center for Eye Disease and Development, \\ Program in Vision Science and School of Optometry, University of California, Berkeley, CA; ${ }^{3}$ Department of Dermatology, University Hospital Zurich, Zurich, \\ Switzerland; ${ }^{4}$ Institute of Immunobiology, Cantonal Hospital St. Gallen, St. Gallen, Switzerland; ${ }^{5}$ Department of Pathology, Albert Einstein College of \\ Medicine, New York, NY; and ${ }^{6}$ Division of Developmental Immunology, Department of Biomedicine, University of Basel, Basel, Switzerland
}

\section{Key Points}

- Afferent lymphatic vessels express interleukin-7.

- Interleukin-7 supports lymphatic drainage.
The cytokine interleukin (IL)-7 exerts essential roles in lymph node (LN) organogenesis and lymphocyte development and homeostasis. Recent studies have identified lymphatic endothelial cells (LECs) as a major source of IL-7 in LNs. Here, we report that LECs not only produce IL-7, but also express the IL-7 receptor chains IL-7R $\alpha$ and CD132. Stimulation with recombinant IL-7 enhanced LEC in vitro activity and induced lymphangiogenesis in the cornea of wild-type (WT) mice. Whereas in IL-7R $\alpha^{-1-}$ mice, dermal lymphatic vessels (LVs) were abnormally organized and lymphatic drainage was compromised, transgenic overexpression of IL-7 in mice resulted in an expanded dermal LV network with increased drainage function. Moreover, systemic treatment with recombinant IL-7 enhanced lymphatic drainage in the skin of WT mice and of mice devoid of lymphocytes. Experiments in IL-7R $\alpha^{-1-}$ bone marrow chimeras demonstrated that the drainage-enhancing activity of IL-7 was exclusively dependent on IL-7R $\alpha$ expression in stromal but not in hematopoietic cells. Finally, near-infrared in vivo imaging performed in IL-7R $\alpha^{-1-}$ mice revealed that the pumping activity of collecting vessels was normal but fluid uptake into lymphatic capillaries was defective. Overall, our data point toward an unexpected new role for IL-7 as a potential autocrine mediator of lymphatic drainage. (Blood. 2013;122(13):2271-2281)

\section{Introduction}

The lymphatic vascular system plays an essential role in tissue fluid homeostasis by absorbing fluid from the interstitium and returning it to the blood circulation. ${ }^{1}$ Lymphatic vessels (LVs) in tissues begin as blind-ended capillaries, which give rise to larger collecting vessels that eventually merge and connect with draining lymph nodes (LNs). In contrast to the absorbing initial lymphatic capillaries, collecting LVs are less permeable, contain valves, and are surrounded by a continuous basement membrane and a smooth muscle cell layer. ${ }^{2}$ The functional units of collecting LVs are the lymphangions, which span between valves and spontaneously contract to propagate lymph and lymph-borne cells. ${ }^{1}$ Besides their role in fluid homeostasis, LVs are highly important for immune function. Whereas afferent LVs mediate the transport of soluble antigen and the migration of leukocytes to draining LNs, efferent LVs contribute to immune surveillance by enabling lymphocyte recirculation between secondary lymphoid organs and the blood. ${ }^{3}$ The intimate connection between the lymphatic vascular system and the immune system is also reflected by the fact that many cytokines that act on immune cells impact lymphatic endothelial cell (LEC) biology. For example, tumor necrosis factor- $\alpha$ upregulates adhesion molecules and inflammatory chemokines in LECs, ${ }^{4,5}$ whereas lymphotoxin ${ }^{6,7}$ and interleukin (IL) $-17^{8}$ have been linked with inflammation-induced

Submitted January 14, 2013; accepted July 26, 2013. Prepublished online as Blood First Edition paper, August 20, 2013; DOI 10.1182/blood-2013-01478073.

M.I. and D.A. contributed equally to this study.

The online version of this article contains a data supplement. lymphangiogenesis. By contrast, interferon- $\gamma^{9}$ and transforming growth factor- $\beta^{10}$ reportedly exert antilymphangiogenic activity.

IL-7 is a homeostatic cytokine produced by stromal cells in primary and secondary lymphoid organs and also by keratinocytes, hepatocytes, and intestinal epithelial cells. ${ }^{11,12}$ Recent reports have shown that besides fibroblastic reticular cells, LN-resident LECs are important producers of IL-7. ${ }^{13-16}$ IL-7 signals through a heterodimeric receptor composed of the IL-7 receptor $\alpha$ chain (IL-7R $\alpha$ or CD127) and the cytokine receptor common $\gamma$ chain (CD132). IL-7 is essential for T- and B-lymphocyte development and supports the survival and homeostatic proliferation of naive and memory T cells. ${ }^{12,17}$ Furthermore, IL-7 plays a central role in LN organogenesis by regulating the activity and number of lymphoid tissue inducer (LTi) cells. ${ }^{12,18,19}$ Mice with defects in IL-7 signaling are characterized by a striking reduction in LN number and LN cellularity. ${ }^{20,21}$ Because of the cytokine's immune-boosting effects, which are mainly attributed to its antiapoptotic and proliferation-inducing activity in T cells, IL-7 therapy is currently under clinical evaluation for the treatment of various forms of cancer and chronic viral infections. ${ }^{12}$

In a microarray study performed on LECs isolated from murine skin, we have recently observed that messages encoding IL-7, IL-7R $\alpha$, and CD132 were present in LECs, ${ }^{5}$ prompting us to further study the

The publication costs of this article were defrayed in part by page charge payment. Therefore, and solely to indicate this fact, this article is hereby marked "advertisement" in accordance with 18 USC section 1734. 
role of these genes in LEC biology. Here, we report that IL-7 and its receptor chains are expressed at the protein level in LECs. In vitro, exogenous as well as endogenous IL-7 supported biologic processes such as LEC tube formation and migration. In vivo, experiments performed in various mouse models, including bone marrow (BM) chimeras, consistently documented the importance of IL-7 and IL-7R $\alpha$ expression in stromal cells in shaping LV morphology and in supporting lymphatic drainage. Overall, our data point toward an autocrine role for IL-7 in the lymphatic vascular system and thus provide novel functional significance for the expression of IL-7 in LECs.

\section{Materials and methods}

\section{Cell culture}

Human LECs and blood vascular endothelial cells (BECs) were isolated from neonatal human foreskins as previously described. ${ }^{22}$ Cells were cultured on plates coated with collagen type I (Advanced BioMatrix, Poway, CA; $3.1 \mathrm{mg} / \mathrm{mL}$ ) and/or fibronectin (Millipore, Billerica, MA; $1 \mathrm{mg} / \mathrm{mL}$ ) in endothelial basal medium (Lonza, Walkersville, MD) supplemented with $20 \%$ fetal bovine serum (GIBCO, Paisley, UK), antibiotic antimycotic solution (1×; Fluka, Buchs, Switzerland), L-glutamine ( $2 \mathrm{mM}$; Fluka), hydrocortisone $\left(10 \mu \mathrm{g} / \mathrm{mL}\right.$; Fluka), and $\mathrm{N}^{6}{ }^{6} 2^{\prime}$-O-dibutyryladenosine $3^{\prime}, 5^{\prime}$-cyclic monophosphate sodium salt $\left(2.5 \times 10^{-2} \mathrm{mg} / \mathrm{mL}\right.$; Fluka). All cells were cultured at $37^{\circ} \mathrm{C}$ in presence of $5 \% \mathrm{CO}_{2}$.

\section{Mice}

C57BL/6 mice were from Charles River Laboratories (Sulzbach, Germany), and $\mathrm{RAG}^{-1-}$ and BALB/c mice were from The Jackson Laboratories (Bar Harbor, ME). IL-7R $\alpha^{-/-}$mice $^{18,20}$ as well as IL-7 transgenic mice expressing murine IL-7 in all cell types ${ }^{23}$ have been previously described. IL-7-CrexR26enhanced yellow fluorescent protein (EYFP) mice $^{16}$ were generated by crossing BAC-transgenic IL-7Cre mice ${ }^{24}$ (kindly provided by Ellen Richie, M. D. Anderson Cancer Center, Houston, TX) with R26-EYFP reporter mice. ${ }^{25}$ All experiments were approved by the Cantonal Veterinary Offices of Zurich and St. Gallen or by the Animal Care and Use Committee of the University of California, Berkeley.

\section{Fluorescence-activated cell sorter (FACS) analysis}

Mouse ear skin or LNs were digested with collagenase IV (Invitrogen, Basel, Switzerland) as previously described. ${ }^{26}$ Single-cell suspensions were stained with anti-mouse CD31-APC, anti-mouse CD45-PerCP (both from Biolegend, San Diego, CA), hamster anti-mouse podoplanin (clone 8.1.1., Developmental Studies Hybridoma-Bank, University of Iowa, IA), and anti-hamster-PE (Invitrogen) or anti-hamster-Alexa 488 (Invitrogen). The expression of IL-7R $\alpha$ and CD132 was detected by additionally staining with anti-mouse CD127FITC (Biolegend), anti-mouse CD132-biotin (eBioscience, San Diego, CA), and streptavidin-Alexa 488 (Invitrogen). IL-7 expression in vivo was analyzed by measuring yellow fluorescent protein (YFP) in ear tissue of IL-7-CrexR26enhance YFP (EYFP) mice. ${ }^{16}$ FACS data were acquired on a BD FACSCanto (BD Biosciences, Allschwil, Switzerland) using FACSDiva software (BD Biosciences). Data were analyzed offline using FlowJo software (Treestar, Ashland, TN).

\section{In vivo systemic treatment with IL-7/anti-IL-7 complexes}

Recombinant human IL-7 (eBioscience) was mixed with the anti-human IL-7 monoclonal antibody clone M25 (NCI BRB Preclinical Repository, Rockville, $\mathrm{MD)}$, as previously described.$^{27}$ Mice received intraperitoneal injections of 1.5 $\mu \mathrm{g}$ of IL-7/anti-IL-7 complexes every other day for a total of 7 days.

\section{Immunofluorescence analysis of ear whole mounts}

Mice were sacrificed and ears harvested after depilation. Ears were split into 2 halves along the cartilage and fixed with $4 \%$ paraformaldehyde for
2 hours. Ears were washed with phosphate-buffered saline (PBS) and incubated for 2 hours in PBS containing 12\% bovine serum albumin, followed by overnight incubations with rabbit anti-mouse lymphatic vessel endothelial hyaluronan receptor (LYVE)-1, rat anti-mouse CD31 (BD Biosciences), or rabbit-anti-YFP (Clontech, Basel, Switzerland). After PBS washes, ears were incubated for $>4$ hours with Alexa-conjugated secondary antibodies (all from Invitrogen). Ears were washed in PBS and fixed for 10 minutes with $4 \%$ paraformaldehyde. Samples were mounted using Vectashield mounting medium (Vector Laboratories, Burlingame, MA) and analyzed on an Zeiss LSM 710-FCS confocal microscope using a $10 \times 0.3$ NA EC Plan-Neofluar and a $20 \times 0.8$ NA Plan-Apochromat objective (Zeiss AG, Goettingen, Germany). Images were acquired using Zeiss ZEN 2009 software (Carl Zeiss AG) and processed using Imaris software (version 7.1.1; Bitplane, Zürich, Switzerland).

\section{Computer-assisted analyses of ear whole mounts}

Images of 3 randomly chosen fields of view from LYVE-1-stained whole mount samples were acquired. Computer-assisted image analysis, including the total LV length and the distance between branching points, was performed using ImageJ. The total area covered by LVs was determined using an areacalculating tool. The average vessel diameter was calculated by dividing the lymphatic surface area with the LV length. The number of branching points was quantified manually.

\section{Lymph flow assessment using Evans blue dye}

A total of $3 \mu \mathrm{L}$ of Evans blue dye (Fluka, $10 \mathrm{mg} / \mathrm{mL}$ in PBS) were injected into the ear of anesthetized mice using a Hamilton syringe. Sixteen hours later, mice were anesthetized and pictures of the ears were taken using a Canon DS126191 camera. Subsequently, mice were sacrificed and Evans blue dye was extracted from the ears by incubation for 3 days in formamide (Fluka) at room temperature. The background-subtracted absorbance was measured on an Infinite M200 microplate reader by measuring the wavelength at 620 and $740 \mathrm{~nm}$. The concentration of dye in the extracts was calculated using a standard curve of Evans blue in formamide.

\section{BM chimeras}

WT or IL-7R $\alpha^{-1-}$ mice were lethally irradiated (950 rad), reconstituted with $5 \times 10^{6} \mathrm{BM}$ cells from either genotype and used in experiments at least 8 weeks after irradiation.

\section{Statistical analysis}

Normally distributed data were analyzed using the Student's $t$ test. Not normally distributed data sets were analyzed using the Mann-Whitney test. Results are presented as mean \pm standard error of the mean (SEM). Differences were considered statistically significant when $P<.05$. Significant outliers, identified by Grubb's test, were excluded. Statistical analysis was performed with Prism 5 (GraphPad, La Jolla, CA).

See supplemental Data on the Blood Web site for quantitative real-time polymerase chain reaction (qPCR) and functional LEC in vitro assays used in this work. See supplemental Material for corneal micropocket assays and near-infrared lymphatic imaging used in this work.

\section{Results}

\section{IL-7 and its receptor chains are expressed by LECs in vivo}

To study the in vivo expression of IL-7 and its receptor chains, LECs and BECs were FACS-sorted from murine ear skin single-cell suspensions (Figure 1A), followed by messenger RNA (mRNA) isolation, generation of amplified complementary DNA, and qPCR analysis. ${ }^{5}$ In this analysis, messages encoding IL-7 and CD132 were considerably higher expressed in LECs compared with BECs; IL-7R $\alpha$ mRNA levels were also slightly increased in LECs over BECs (Figure 1B). FACS 

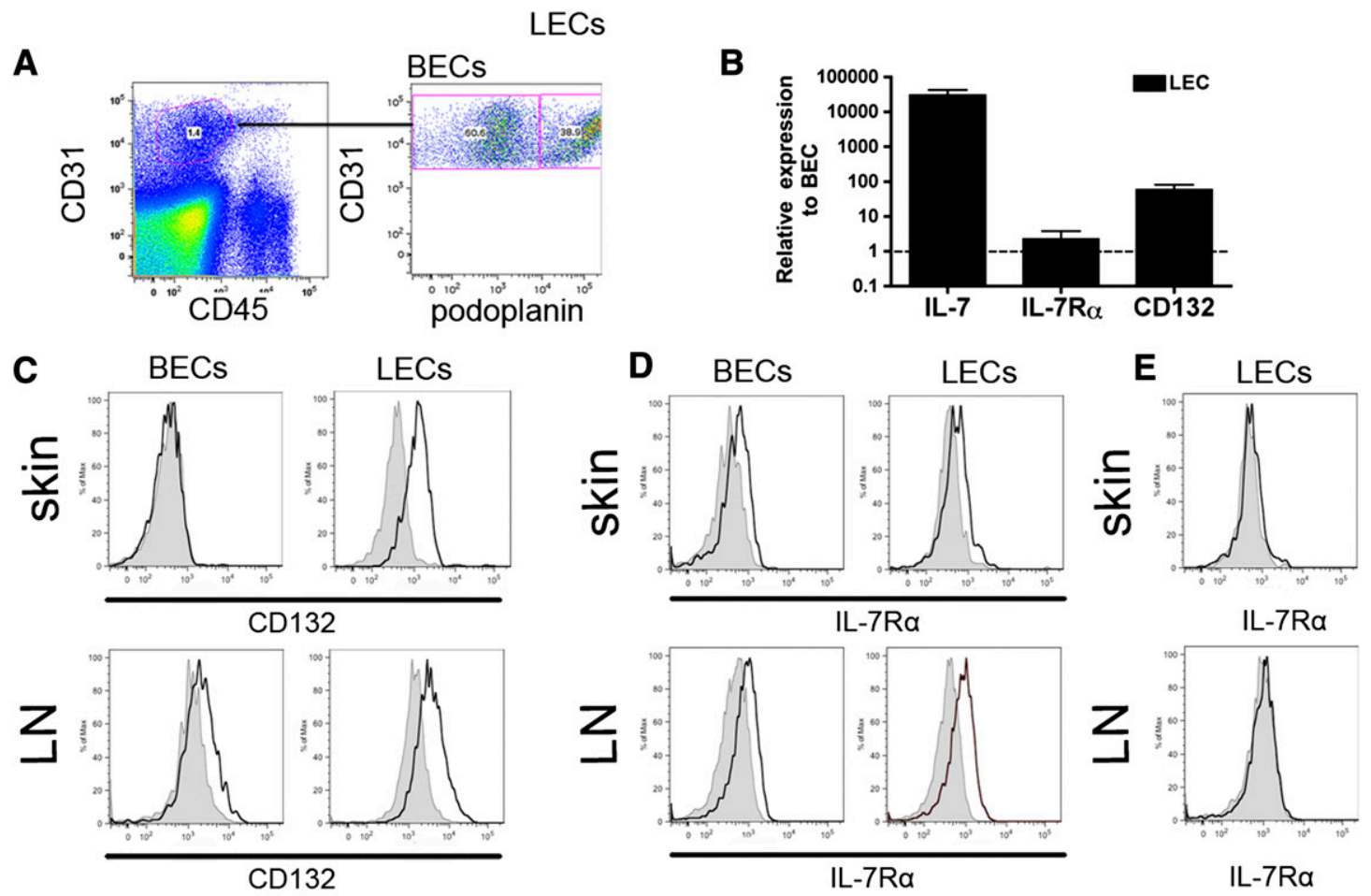

$\mathbf{F}$
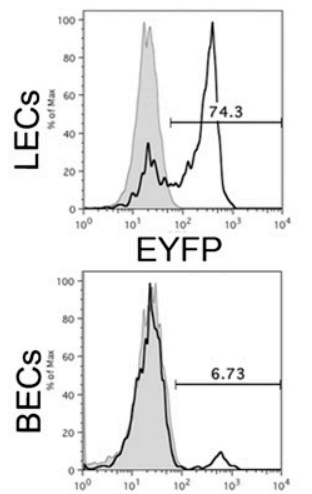

EYFP

G

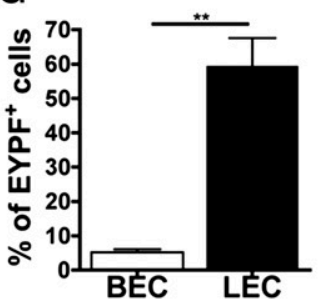

H

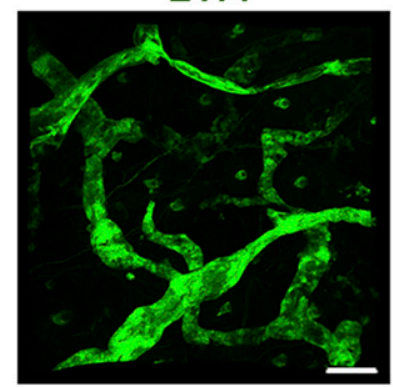

EYFP

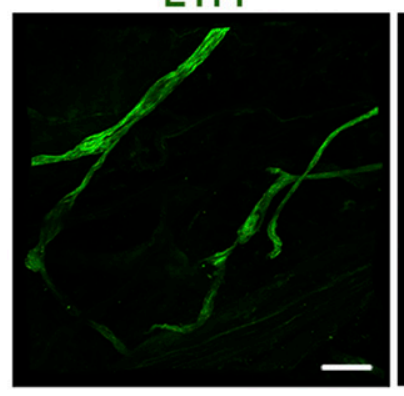

\section{LYVE-1}

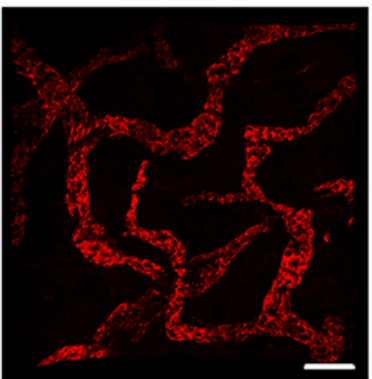

MECA-32

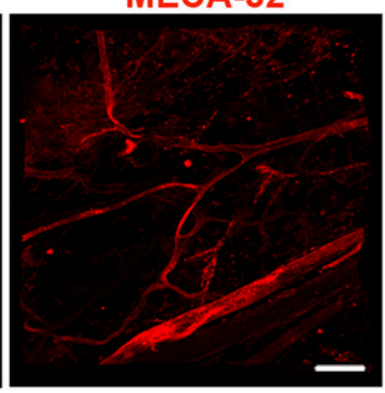

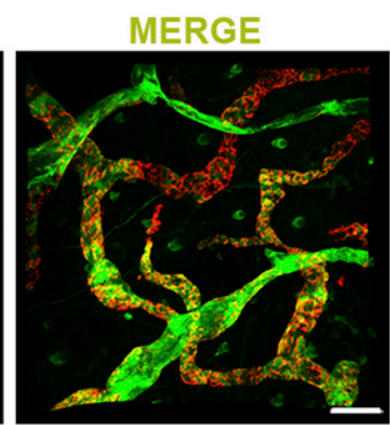

MERGE

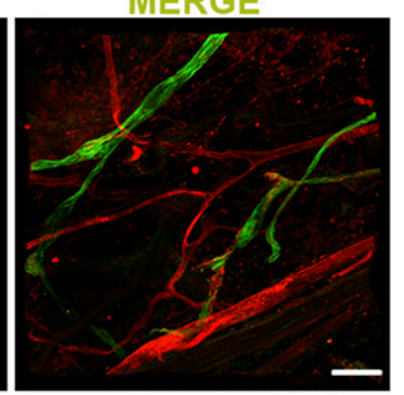

Figure 1. IL-7 and its receptor chains are expressed in LECs in vivo. (A-B) FACS sorting was used to isolate LECs and BECs from single-cell suspensions generated from enzymatically digested mouse ear skin. qPCR was performed on the extracted, reversely transcribed and amplified complementary DNA material. (A) Depiction of the gating scheme used to isolate BECs (CD45 ${ }^{-}$CD $31^{+}$podoplanin ${ }^{-}$) and LECs (CD45 ${ }^{-}$CD $31^{+}$podoplanin ${ }^{+}$). (B) qPCR analysis revealed that IL-7 and its receptor chains were more highly expressed in LECs compared with BECs. Pooled data from 3 experiments are shown. (C-E) To validate the expression of candidate genes in LECs (gated on CD45 CD $31^{+}$podoplanin ${ }^{+}$cells) and BECs (gated on CD $45^{-}$CD $31^{+}$podoplanin ${ }^{-}$cells) at the protein level, FACS analysis was performed on ear and brachial LN single-cell suspensions. (C) CD132 was preferentially expressed in LECs compared with BECs, whereas (D) IL-7R $\alpha$ was similarly expressed in LECs and BECs. (E) No expression of IL7R $\alpha$ was detected when performing FACS analysis on ear skin and LN single-cell suspensions from IL-7R $\alpha^{-1-}$ mice. Representative plots from 3 different experiments are shown. (F) FACS analysis performed on single-cell suspensions generated from the ears of IL-7-CrexR26-EYFP mice confirmed YFP expression in most LECs (gated on CD $45^{-}$CD $31^{+}$podoplanin ${ }^{+}$), but not in BECs (gated on CD45 ${ }^{-}$D $31^{+}$podoplanin ${ }^{-}$). Representative FACS plots showing YFP expression in LECs and BECs are shown. (G) A summary of the percentages of YFP-expressing LECs and BECs from all experiments $(n=3)$ is shown. (H-I) Confocal laser scanning microscopic analysis of ear skin whole mounts prepared from IL-7-CrexR26-EYFP mice detected YFP expression in virtually all LYVE- $1^{+}$LVs $(H)$, but not in MECA-32 ${ }^{+}$blood vessels $(\mathrm{I})$. Scale bar represents $100 \mu \mathrm{m}$. ${ }^{\star \star} P<.01$. 
A

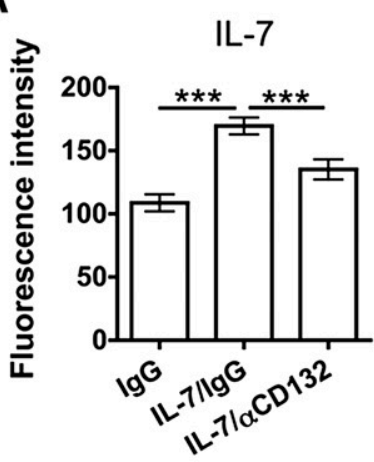

C

\section{CTR}

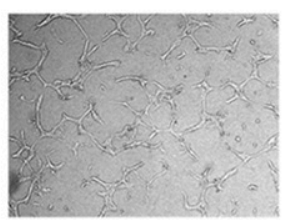

$\mathbf{E}$

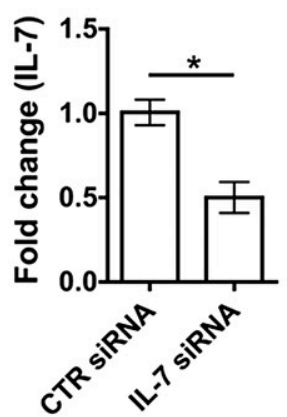

H

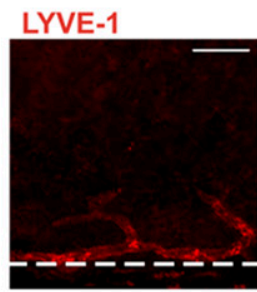

CTR

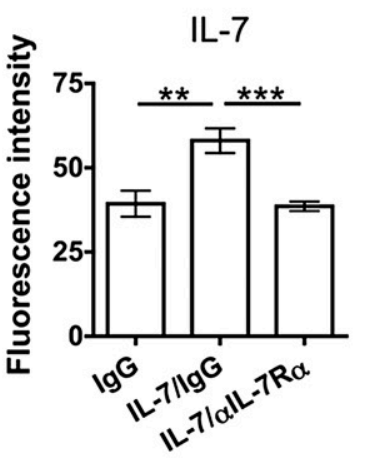

D
B
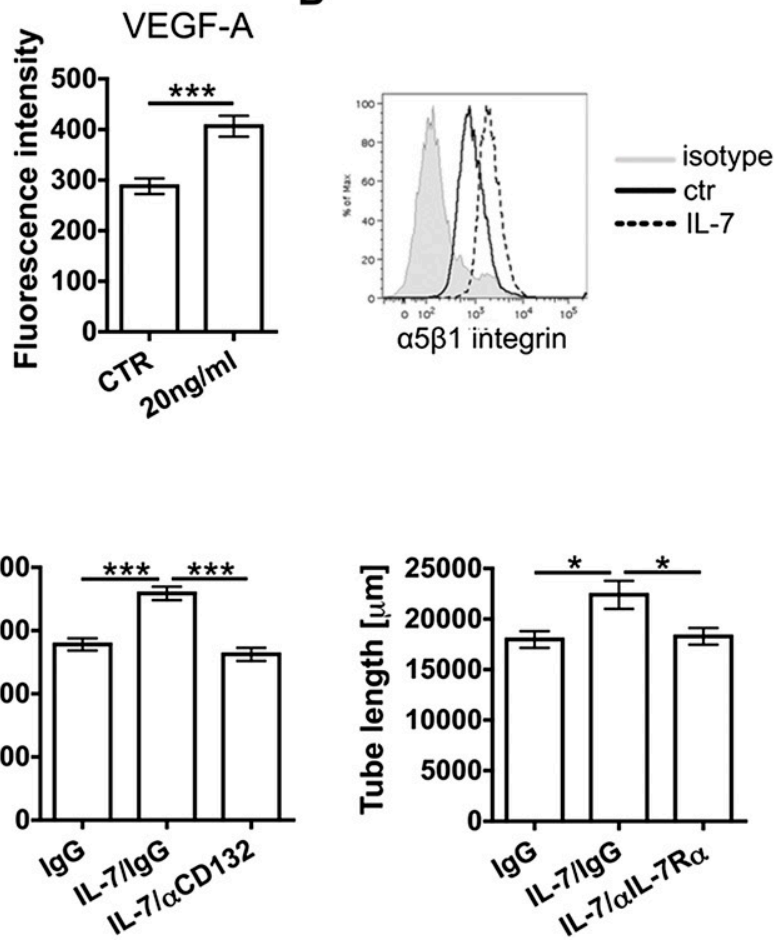

G

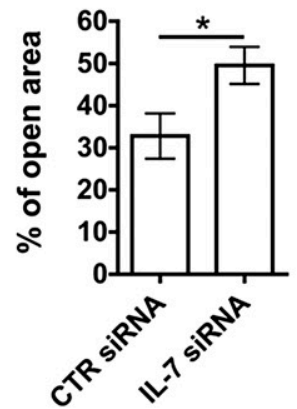

Figure 2. IL-7 activates LEC in vitro function and induces lymphangiogenesis in vivo. (A-G) Functional in vitro assays were performed to analyze the effect of IL-7 on LECs. (A) Overnight treatment with IL-7 significantly enhanced LEC adhesion to fibronectin-coated plates. This response could be reversed by concomitant treatment with CD132 or IL-7R $\alpha$ blocking antibodies. Notably, the IL-7-induced response was comparable to the one induced by treatment with VEGF-A (positive control). (B) Overnight treatment of LECs with IL-7 led to an upregulation of the fibronectin-binding integrin $\alpha_{5} \beta_{1}$, as seen in FACS. Data from 1 of 4 similar experiments are shown. (C-D) Tube formation assays were performed by covering confluent LEC monolayers with collagen type I containing IL-7 (50 ng/mL). (C) Representative images showing the IL-7-induced increase in LEC tube formation. (D) IL-7-induced tube formation could be reversed when performing the assay in presence of CD132 or IL-7R $\alpha$ blocking antibodies. (E-G) Effect of siRNA-mediated knockdown of IL-7 in LECs. (E) Transfection with siRNA significantly reduced IL-7 mRNA levels in LECs (E) as well as the capacity of LECs (F) to form tube-like structures. (G) Moreover, knockdown of IL-7 levels in LECs reduced LEC migration, as measured in a scratch-wound assay. Data from 1 of 3 similar experiments are shown. (H-I) To analyze the effect of IL-7 on lymphangiogenesis in vivo, a cornea micropocket assay was performed. (H) Representative immunofluorescent micrographs demonstrating ingrowth of new LVs into the cornea 14 days after IL-7 pellet implantation. LYVE-1: red. Scale bar represents $50 \mu \mathrm{m}$. (I) A significant difference was observed in the area covered by lymphatics between the treatment and the control group (n $=9$ mice per group). ${ }^{\star} P<.05 ;{ }^{\star \star} P<.01 ;{ }^{\star \star \star} P<.001$. ctr, control; IgG, immunoglobulin G. 
Figure 3. The LV network and lymphatic drainage are compromised in IL-7R $\boldsymbol{\alpha}^{-1-}$ mice. (A-E) The LYVE-1 ${ }^{+}$ lymphatic network in the ear skin of IL-7R $\alpha^{-1-}$ and WT control mice was analyzed in whole mount preparations. (A) Representative images showing LYVE-1+ $\mathrm{LVs}$ in the ear skin of both genotypes. Scale bar represents $200 \mu \mathrm{m}$. (B-E) Image-based morphometric analysis revealed a significant decrease (B) in average LYVE-1 $1^{+}$vessel diameter, whereas the average LYVE- $1^{+}$area $(C)$ was increased in the ear skin of IL-7R $\alpha^{-1-}$ compared with WT mice. (D) The number of branching points was significantly increased, whereas the $(\mathrm{E})$ distance between branching points was decreased in IL-7R $\alpha^{-1-}$ compared with WT mice. (F-G) To evaluate lymphatic drainage function, Evans blue dye was intradermally injected into the ears of IL-7R $\alpha^{-/-}$and WT mice, and the dye content remaining in the ear was extracted and quantified 16 hours later. (F) Quantification detected increased Evans blue levels in the ears of IL-7R $\alpha^{-1-}$ mice, indicative of defective lymphatic drainage. (G) Representative pictures taken 16 hours after Evans blue injection. Pooled data from 2 similar experiments involving a total of 10 mice/group are shown. ${ }^{\star} P<.05$; ${ }^{\star \star} P<.01 ;{ }^{\star \star \star} P<.001$
A

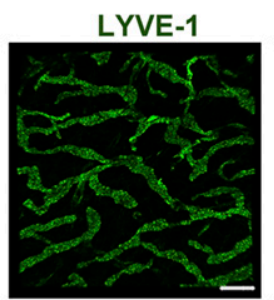
WT

C

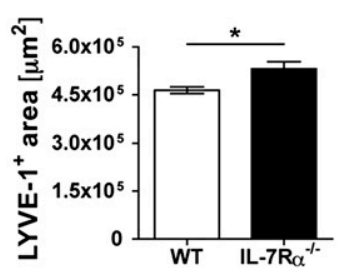

LYVE-1

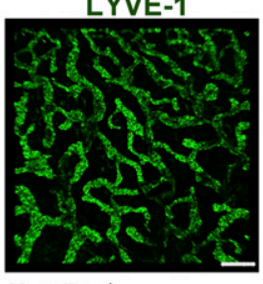

IL-7Ra ${ }^{-1-}$
B

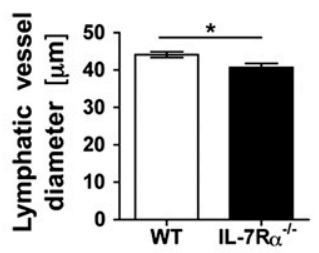

E

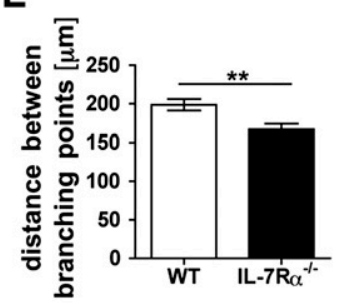

$\mathbf{F}$

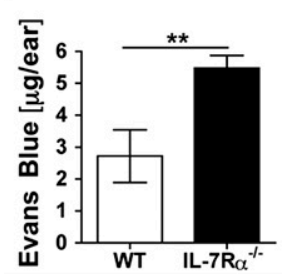

G

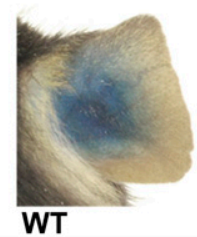

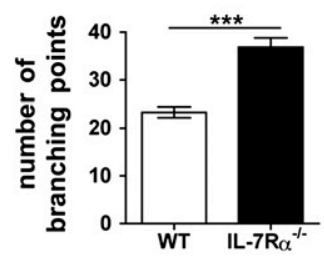

analysis performed on ear skin and LN single-cell suspensions confirmed higher expression of CD132 protein in LECs compared with BECs (Figure 1C), whereas IL-7R $\alpha$ protein levels were comparable (Figure 1D). Importantly, no signal for IL-7R $\alpha$ was detected when analyzing skin or brachial LN LECs from IL-7R $\alpha^{-1-}$ mice (Figure 1E). To further assess whether dermal LECs produce IL-7, we analyzed the skin of IL-7-CrexR26-EYFP mice, ${ }^{16}$ which express EYFP in cells with past or present IL-7 promoter activity. ${ }^{16}$ FACS analysis of ear skin single-cell suspensions revealed that the majority of LECs but virtually no BECs were EYFP ${ }^{+}$(Figure 1F-G). Similarly, whole mount analyses performed in the ear skin of IL-7-CrexR26-EYFP mice detected EYFP expression in virtually all LYVE- $1^{+}$LVs, but not in MECA $32^{+}$blood vessels (Figure 1H-I). IL-7 expression was also detected in in vitro-cultured murine dermal $\mathrm{LECs}^{5}$ isolated from Immortomice ${ }^{28}$ (data not shown). In vitro-cultured murine dermal LEC supernatant significantly enhanced the survival of murine $\mathrm{CD}^{+} \mathrm{T}$ cells, and this survival-enhancing activity was reduced upon addition of IL-7blocking antibody (supplemental Figure 1), indicating IL-7 bioactivity. Notably, similar results were recently obtained for IL-7 derived from LN LECs. ${ }^{16}$ Overall, our findings revealed that murine dermal LECs expressed both functional IL-7 and the IL-7 receptor chains.

\section{IL-7 activates LECs in vitro and induces lymphangiogenesis in vivo}

To further investigate the function of IL-7 and its receptor chains in LECs, we performed in vitro studies with primary dermal human LECs, which expressed detectable levels of IL-7, IL-7R $\alpha$, and CD132 mRNA and protein (supplemental Figure 2). IL-7 was previously shown to induce lymphatic marker gene expression and tube formation in a human endothelial cell line. ${ }^{29}$ To analyze whether stimulation with exogenous IL-7 would induce similar cellular responses in primary human LECs, functional assays were performed. IL-7 treatment induced only weak proliferation in LECs (data not shown), but led to a strong increase in LEC adhesion to fibronectin (Figure 2A). This effect could be reversed by addition of antibodies blocking CD132 or IL-7R $\alpha$ and was comparable to the effect induced by vascular endothelial growth factor-A (VEGF-A) treatment (Figure 2A). Increased adhesion to fibronectin was paralleled by an IL-7-induced upregulation of the fibronectin-binding integrin $\alpha_{5} \beta_{1}{ }^{30}$ (Figure 2B). IL-7 treatment also induced a dose-dependent increase in LEC migration in a scratch-wound assay (supplemental Figure 3A-B) and a striking upregulation of the lymphatic-specific transcription factor Prox- $1^{31}$ (supplemental Figure 3C-F). Moreover, stimulation with exogenous IL-7 significantly enhanced the ability of cultured LECs to form tube-like structures in a collagen matrix (Figure 2C-D). This process was reversed when performing the assay in the presence of CD132- or IL-7R $\alpha$-blocking antibodies (Figure 2D). Interestingly, small interfering RNA (siRNA)-mediated knockdown of IL-7 in LECs significantly reduced LEC tube formation and migration (Figure 2E-G), suggesting an autocrine contribution of IL-7 to LEC biology. Although incubation of LECs with IL-7 only induced weak phosphorylation of the signal transducer and activator of transcription (Stat)5, a well-known mediator of IL-7 signaling in hematopoietic cells, ${ }^{17}$ treatment with a Stat5-inhibitor completely reverted IL-7-induced tube formation (supplemental Figure 3G-H). To investigate the lymphangiogenic properties of IL-7 in vivo, a containing either IL-7 or vehicle control were surgically implanted into the corneas of wild-type (WT) mice and the growth of vessels toward the pellet was analyzed 14 days later by immunofluorescence. IL-7-containing pellets induced a significant lymphangiogenic response in the cornea (Figure 2H-I). In conclusion, our findings identified IL-7 as a potent inducer of lymphangiogenesis in vitro and in vivo. mouse corneal micropocket assay was performed. For this, pellets 
A

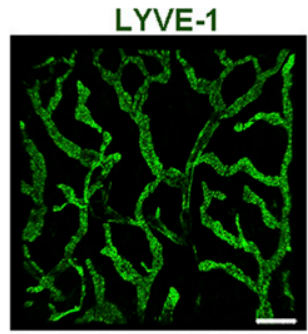

$$
\text { WT }
$$

C

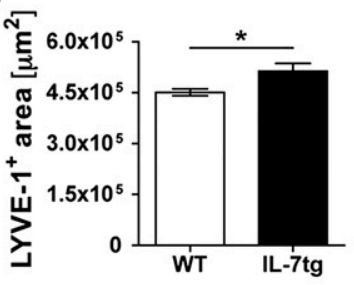

$\mathbf{F}$

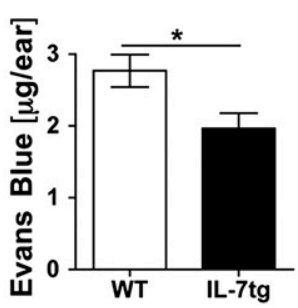

LYVE-1

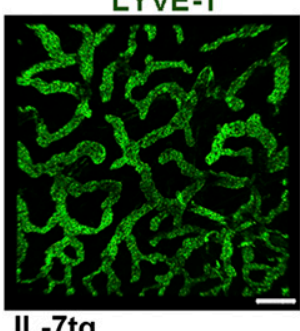

D

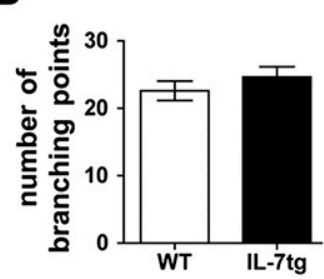

B

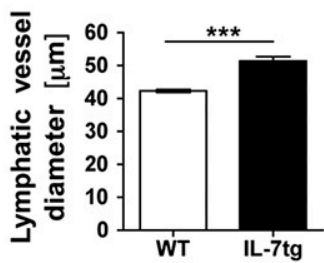

E

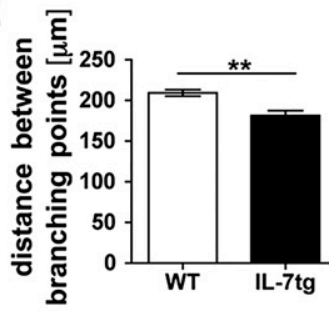

Figure 4. The LV network is expanded and displays enhanced drainage in the skin of IL-7 transgenic mice. (A-E) The LYVE-1 ${ }^{+}$Iymphatic network in the ear skin of transgenic mice expressing IL-7 in all cells (IL-7tg) or in WT control mice was analyzed in whole mount preparations. (A) Representative images showing LYVE $-1^{+}$LVs in the ear skin of both genotypes. Scale bar represents $200 \mu \mathrm{m}$. (B-E) Image-based morphometric analysis revealed that the average LV diameter $(\mathrm{B})$ and the average LYVE-1 $1^{+}$area (C) were significantly increased in ear skin of IL-7tg compared with WT mice. (D) The number of branching points remained unchanged, whereas the average distance between branching points (E) was reduced in IL-7tg compared with WT mice. Results from 1 of 2 similar experiments are shown $(n=4$ mice/group). (F-G) To evaluate lymphatic drainage function, Evans blue dye was intradermally injected into the ears of IL-7tg and WT mice and the dye content remaining in the ear was extracted and quantified 16 hours later. (F) Quantification revealed reduced Evans blue levels in the ears of IL-7tg mice, indicative of increased lymphatic drainage. (G) Representative pictures taken 16 hours after Evans blue injection. Pooled data from 3 experiments involving a total of 15-18 mice/group are shown. ${ }^{\star} P<.05 ;{ }^{* *} P<.01 ;{ }^{* \star} P<.001$.

G

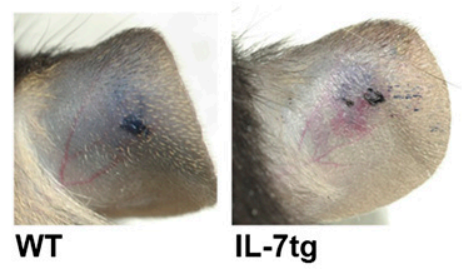

Impaired drainage function of dermal lymphatics in IL-7R $\boldsymbol{\alpha}^{-l-}$ mice

We next analyzed the LV phenotype in IL-7R $\alpha^{-1-}$ mice. Immunofluorescent staining of ear skin whole mounts for the lymphatic capillary marker LYVE- $1^{2}$ revealed striking differences in the organization of the LV network between IL-7R $\alpha^{-1-}$ and WT mice (Figure 3A). LV diameters were significantly reduced (Figure 3B) and the lymphatic network in IL-7R $\alpha^{-1-}$ mice was more dense, as evidenced by an increase in the total area covered by LVs (Figure 3C), a strong increase in the number of branching points (Figure 3D), and a reduced distance between branching points (Figure 3E). To investigate whether these morphologic abnormalities had any functional consequences, we performed lymphatic drainage experiments by injecting small amounts of Evans blue into the ear pinna. Evans blue, which rapidly binds albumin in situ, is specifically taken up by lymphatics upon intradermal injection. ${ }^{32,33}$ When analyzing the ears 16 hours after injection, approximately 2-fold more Evans blue was retained in the ears of IL-7R $\alpha^{-1-}$ mice compared with WT mice (Figure 3F-G), indicating decreased drainage function. Notably, when performing the same experiment in K14-VEGFR3-Ig mice, which completely lack dermal lymphatic capillaries and mount a severe drainage phenotype, ${ }^{34}$ a 4.5 -fold increase in Evans blue retention in the ears was measured (supplemental Figure 4). Thus, the 2-fold difference observed in IL-7 $\mathrm{R} \alpha^{-1-}$ mice, which still retain an-albeit disorganized-dermal lymphatic capillary network (Figure 3A-E), suggested a substantial defect in drainage.

\section{Enhanced drainage function of the dermal lymphatics in IL-7tg mice}

We next assessed the lymphatic phenotype and function in transgenic mice ubiquitously overexpressing IL-7 in all cells types (IL-7tg). ${ }^{23}$
Here, the diameters of dermal LVs as well as the area covered by LVs were significantly increased in comparison with WT control (Figure 4A-C). By contrast, the number of branching points was similar, whereas the distance between branching points was reduced in IL-7tg compared with WT mice (Figure 4D-E). Performing Evans blue drainage experiments approximately $30 \%$ less dye was retained in the ears of IL-7tg compared with WT mice 16 hours after injection (Figure 4F-G). Thus, whereas loss of IL-7 receptor signaling reduced lymphatic drainage (Figure 3F-G), transgenic overexpression of IL-7 had the opposite effect.

\section{Systemic treatment with recombinant IL-7 enhances lymphatic drainage in the skin}

Our studies in IL-7 R $\alpha^{-1-}$ and IL-7tg mice indicated that IL-7 signaling supported lymphatic drainage function. However, it remained unclear whether the effects observed were caused by genotype-induced developmental differences in the lymphatic network or whether IL-7 remained important for lymphatic drainage function in adulthood. To address this question, adult WT mice were injected intraperitoneally for 7 days with recombinant IL-7 complexed with anti-IL-7 antibody (IL-7/anti-IL-7 complexes) (Figure 5A). IL-7/anti-IL-7 complexes represent a well-documented method for boosting the in vivo efficacy of IL-7, presumably by enhancing IL-7 pharmacokinetics and half-life. ${ }^{18,27}$ Treatment with IL-7/anti-IL-7 complexes induced a strong lymphoproliferative response in the spleen of WT mice (data not shown). Drainage experiments revealed that complex treatment significantly enhanced lymphatic clearance of Evans blue from the injected ear (Figure 5B-C). IL-7/anti-IL-7 complex treatment also enhanced dermal lymphatic drainage in $\mathrm{RAG}^{-1-}$ mice lacking $\mathrm{T}$ and B lymphocytes (Figure 5D-E). Moreover, complex treatment induced some subtle, near-significant morphologic changes in dermal LVs in WT and $\mathrm{RAG}^{-/-}$mice (supplemental Figure 5). Thus, 
Figure 5. Systemic treatment with recombinant IL-7/ anti-IL-7 complexes equally enhances lymphatic drainage in the skin or WT and of RAG1 ${ }^{-1-}$ mice. (A) $\mathrm{WT}$ and $\mathrm{RAG} 1^{-1-}$ mice were treated intraperitoneally every other day for a total of 7 days with IL-7/anti-IL-7 complexes or PBS. On day 6, Evans blue dye was injected intradermally into the ears of mice. Animals were sacrificed on day 7 and ears were used for Evans blue quantification. (B-C) IL-7/anti-IL-7 complextreated WT mice exhibited increased drainage function of dermal LVs upon intradermal injection of Evans blue. (B) Quantification of the total Evans blue dye present in the ear 16 hours after injection. (C) Representative pictures taken 16 hours after Evans blue injection. (D-E) IL-7/anti-IL-7 complex-treated RAG1 ${ }^{-1-}$ mice exhibited increased drainage function of dermal LVs upon intradermal injection of Evans blue. (D) Quantification of the total Evans blue dye present in the ear 16 hours after injection. (E) Representative pictures taken 16 hours after Evans blue injection. Pooled data from a total of 10 mice/group are shown. ${ }^{\star \star} P<.01$.
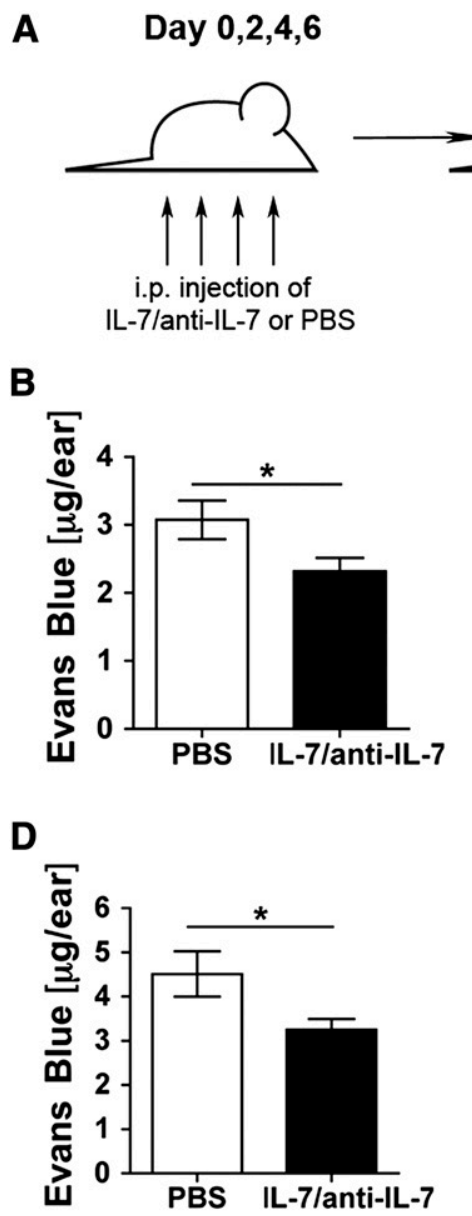

Day $6 \quad$ Day 7

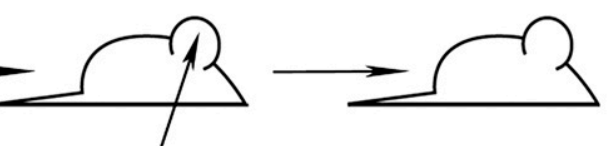

i.d. Evans Blue injection into the ear

Analysis of lymphatic network organization and drainage

C
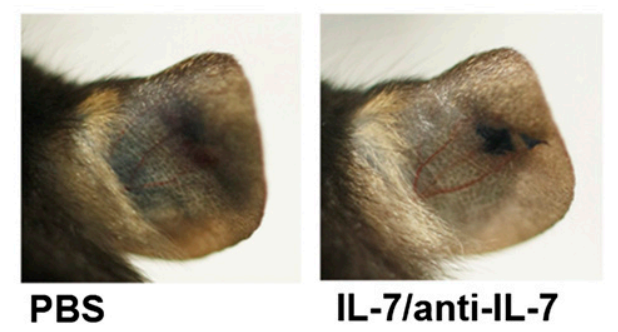

$\mathbf{E}$
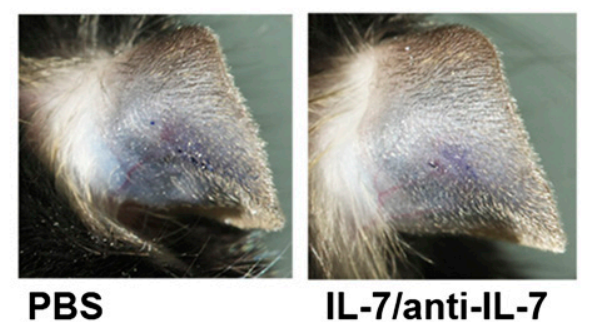

administration of IL-7 in a therapy-like setting was capable of enhancing LV drainage function in mice.

\section{Normal lymphatic morphology and drainage depends on IL-7R $\alpha$ expression in stromal cells}

The results obtained in $\mathrm{RAG}^{-/-}$mice demonstrated that the drainageenhancing activity of recombinant IL-7 did not depend on IL-7responsive $\mathrm{T}$ and $\mathrm{B}$ lymphocytes (Figure 5D-E). However, IL-7 also induces important biologic responses in other hematopoietic cells, such as in LTi cells or dendritic cells. ${ }^{12,19}$ To further investigate the contribution of hematopoietic vs stromal cell-expressed IL-7R $\alpha$ to the lymphatic phenotype and drainage function, BM chimeras were generated. Interestingly, analysis of the ear thickness revealed that the ears of IL-7R $\alpha^{-1-}$ mice reconstituted with either WT or IL$7 \mathrm{R} \alpha^{-1-} \mathrm{BM}$ were significantly thicker compared with those of WT mice reconstituted with either BM (supplemental Figure 6A). As expected, the LN cellularity was reduced in BM chimeras reconstituted with IL-7R $\alpha^{-/-}$BM (Figure 6A). Moreover, whole mount analysis performed in the diaphragm revealed that the abnormal lymphatic phenotype observed in IL-7 R $\alpha^{-/-}$mice (Figure 3A-E) was recapitulated in BM chimeras with IL-7R $\alpha^{-l-}$ stromal cells, whereas loss of IL-7R $\alpha$ in hematopoietic cells had no effect (supplemental Figure 6B-D). Finally, Evans blue experiments demonstrated that drainage was significantly reduced in chimeras with IL-7R $\alpha^{-/-}$ stromal cells, independent of whether mice had been reconstituted with WT or IL-7R $\alpha^{-1-}$ BM (Figure 6B; supplemental Figure 6E). Conversely, no defect in drainage was observed in WT mice that had been reconstituted with IL-7R $\alpha^{-/-}$BM compared with WT mice reconstituted with WT BM (Figure 6B). Notably, the drainage phenotype observed in $\mathrm{BM}$ chimeras (Figure 6B) was less pronounced than in IL-7 $\alpha^{-/-}$mice (Figure 3F-G), possibly because of general irradiation-induced lymphatic dysfunction ${ }^{35}$ or the advanced age of the chimeras. In line with our previous findings, treatment with IL-7/ anti-IL-7 complexes enhanced lymphatic drainage in BM chimeras with functional IL-7R $\alpha$ in stromal cells, irrespective of IL-7R $\alpha$ expression in hematopoietic cells (Figure 6C-D). Notably, no enhancement of drainage was observed in chimeras expressing IL-7R $\alpha$ in hematopoietic but not in stromal cells (Figure 6D). Thus, the drainagepromoting activity of IL-7 was exclusively dependent on IL-7R $\alpha$ expression in stromal cells, suggesting a direct effect of IL-7 on LECs.

\section{IL-7R $\alpha^{-1-}$ mice display a defect in fluid uptake and transport in lymphatic capillaries}

To further investigate the mechanism of reduced lymphatic drainage in IL-7R $\alpha^{-1-}$ mice, near-infrared in vivo lymphatic imaging was performed. To this end, a near-infrared lymphatic tracer was injected into the hind paw and the lower limb was dynamically imaged under a stereomicroscope. This analysis detected no significant difference in the morphology or the pulse rates of the collecting LVs in IL-7R $\alpha^{-1-}$ compared with WT mice (Figure 7A-C; supplemental Videos 1 and 2). Instead, lymphatic dysfunction was observed at the level of the initial lymphatic capillaries. Although tracer uptake was normal in most WT mice, with only deeper collecting vessels visible proximal to the injection site, the majority of IL-7R $\alpha^{-/-}$mice displayed increased spreading 
A

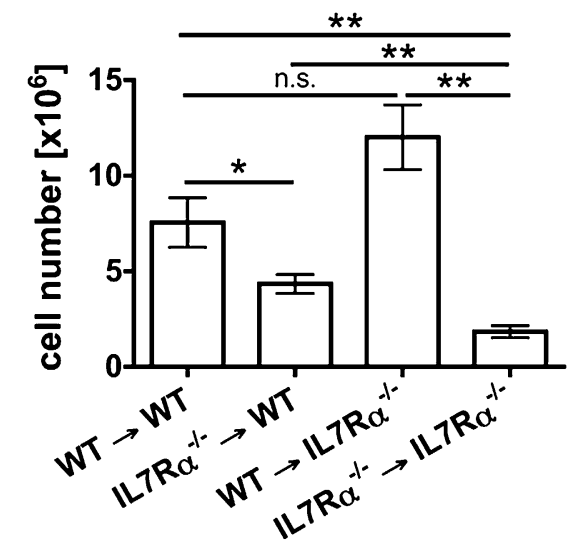

C
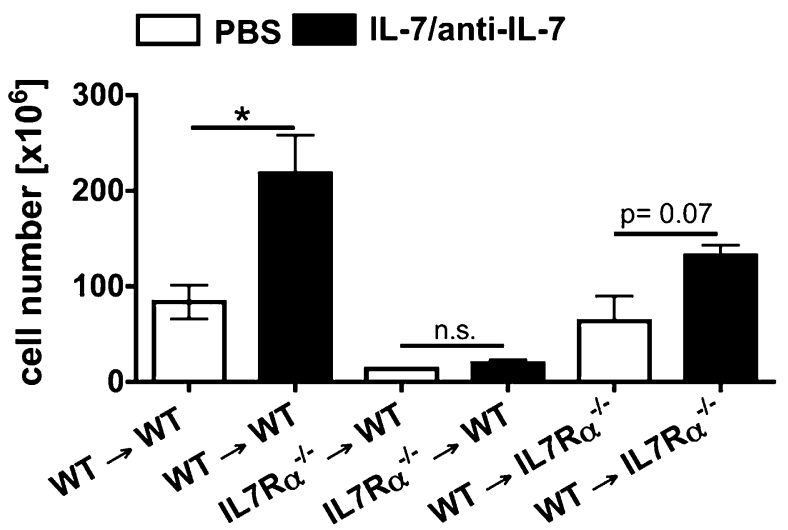

B

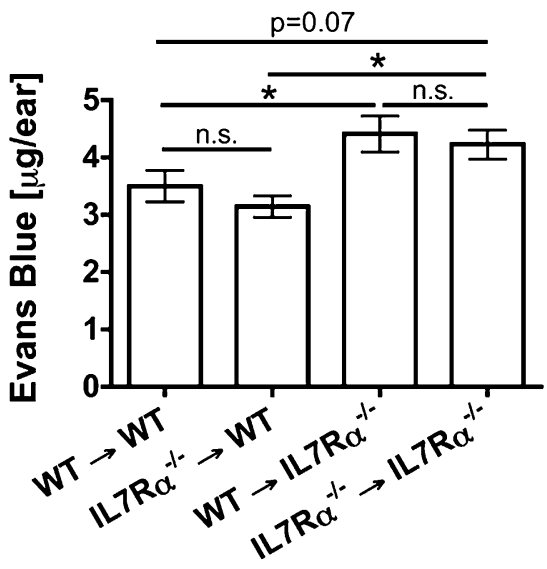

D

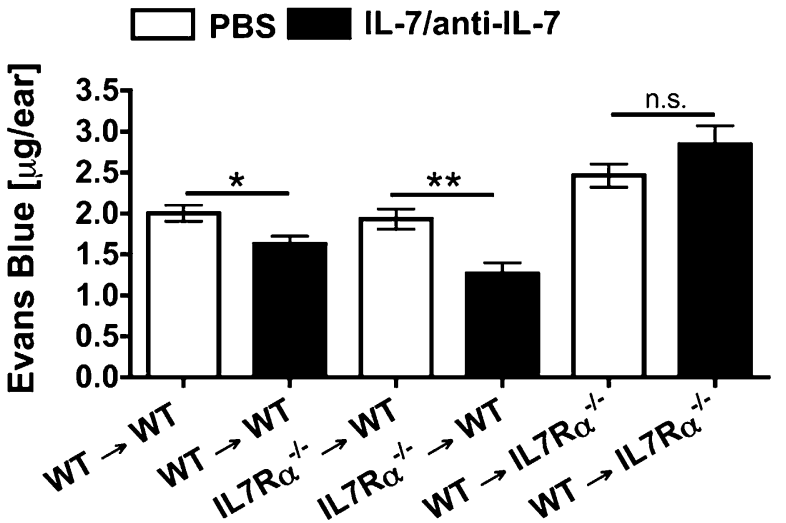

Figure 6. Intact lymphatic drainage and the drainage-promoting activity of IL-7 depend on IL-7R $\alpha$ expression in stromal cells. BM chimeras between WT and IL-7R ${ }^{-1-}$ mice were generated and directly analyzed or subjected to IL-7/anti-IL-7 complex treatment. (A-B) Analysis of BM chimeras. (A) The LN cellularity was reduced in BM chimeras reconstituted with IL-7R $\alpha^{-1-}$ BM. Bars represent pooled data from 2 inguinal and 2 brachial LNs per mouse. (B) To evaluate lymphatic drainage function, Evans blue dye was intradermally injected into the ears of BM chimeras and the dye content remaining in the ear was extracted and quantified 16 hours later. Quantification detected increased Evans blue levels in the ears of BM chimeras with IL-7R $\alpha^{-1-}$ stromal cells, indicative of defective lymphatic drainage. (C-D) Treatment of BM chimeras with IL-7/anti-IL-7 complexes. (C) IL-7/anti-IL-7 complex treatment led to a significant increase in the splenic cellularity of chimeras reconstituted with WT but not with IL-7R $\alpha^{-1-}$ BM. (D) Evans blue drainage experiments revealed that treatment of BM chimeras with IL-7/anti-IL-7 complexes only enhanced lymphatic drainage in chimeras with functional IL-7R $\alpha$ in stromal cells $\left(\mathrm{WT} \rightarrow \mathrm{WT}\right.$; IL-7R $\left.\alpha^{-1-} \rightarrow \mathrm{WT}\right)$. Complex treatment of chimeras exclusively expressing IL-7R $\alpha$ in hematopoietic cells but not in stromal cells $\left(\mathrm{WT} \rightarrow \mathrm{IL}-7 \mathrm{R} \alpha^{-1-}\right.$ ) did not improve drainage. Pooled data from 1-2 experiments involving 4-6 mice/group are shown. ${ }^{\star} P<.05 ;{ }^{\star \star} P<.01$. n.s., not significant.

of the tracer through superficial initial lymphatic capillaries, with evidence of leakage of the tracer into the interstitium (Figure 7D-E).

\section{Discussion}

In this paper, we have analyzed the biologic role of IL-7 in the lymphatic system and identified a previously unknown activity of this cytokine in promoting lymphatic drainage. Several studies have recently described the expression of IL-7 by LECs in LNs. ${ }^{13-16} \mathrm{We}$ now report that LECs isolated from human or murine skin as well as LECs also present in murine skin produce this cytokine. Together with very recent data from IL-7 knock-in mice, ${ }^{15,36}$ our findings indicate that LECs might be a source of IL-7 throughout the body. Interestingly, we also detected IL-7 protein in human skin-draining afferent lymph ${ }^{37}$ (supplemental Figure 7). However, given that IL-7 is also expressed by dermal keratinocytes (supplemental Figure 2), ${ }^{11,12}$ LECs are likely not the only source of IL-7 in skin-draining afferent lymph. The IL-7 levels detected in lymph (15-35 pg/mL) were similar to values detected in blood (supplemental Figure 7$)^{38}$ and are in the range of IL-7 concentrations shown to elicit biologic responses in $\mathrm{T}$ cells in vitro. ${ }^{13,16}$ By contrast, the IL-7 concentrations required to achieve biologic responses in cultured LECs where considerably higher $(>5 \mathrm{ng} / \mathrm{mL})$. This indicates that lymph-borne IL-7 might not primarily act in a paracrine manner on LECs. More likely, LECs consume their self-synthesized IL-7, which likely reaches much higher local concentrations in vicinity of the secreting cell. Our hypothesis that IL-7 might act in an autocrine manner is also supported by our in vitro findings demonstrating reduced tube formation and migration upon siRNA-mediated knockdown of IL-7 in LECs. Moreover, treatment of RAG1 ${ }^{-/-}$or BM chimeras with IL-7/anti-IL-7 complexes demonstrated that the drainage-promoting activity of IL-7 required IL-7R $\alpha$ expression in stromal but not in hematopoietic cells. The latter findings exclude any contribution of IL-7-responsive lymphocytes or LTi cells to the drainage enhancing activity of IL-7 and rather suggest a direct effect of IL-7 on IL-7 receptor-expressing LECs. Clearly, the ultimate proof of this new concept of an autocrine role of IL-7 in LEC biology will require further experiments in mice harboring an LEC-specific deletion of IL-7 or IL-7R $\alpha$. In fact, the tools for generating such mice have recently been generated. ${ }^{39-42}$ 
A

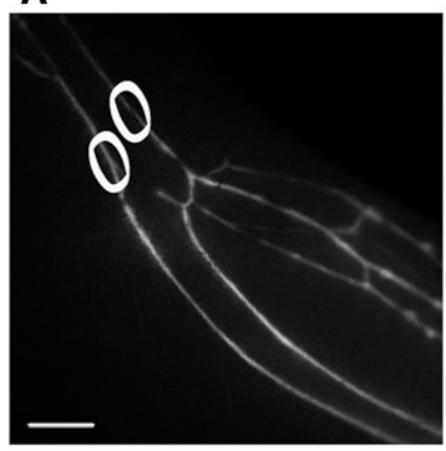

WT

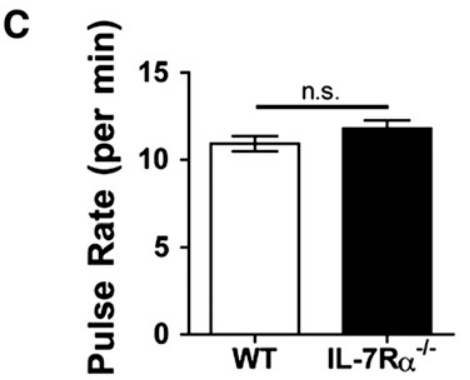

D

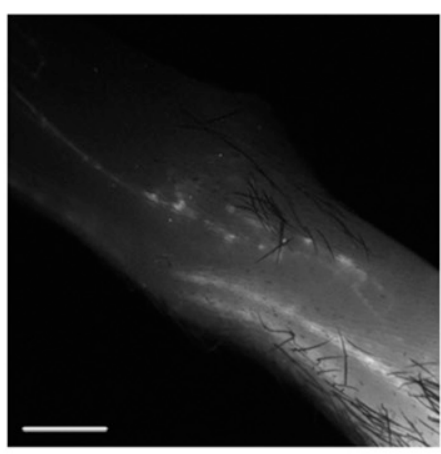

normal

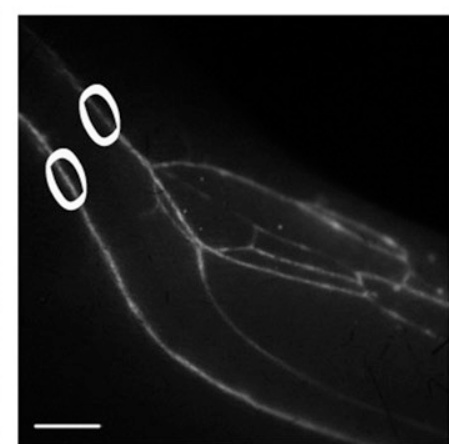

IL-7R $\alpha^{-1-}$

B
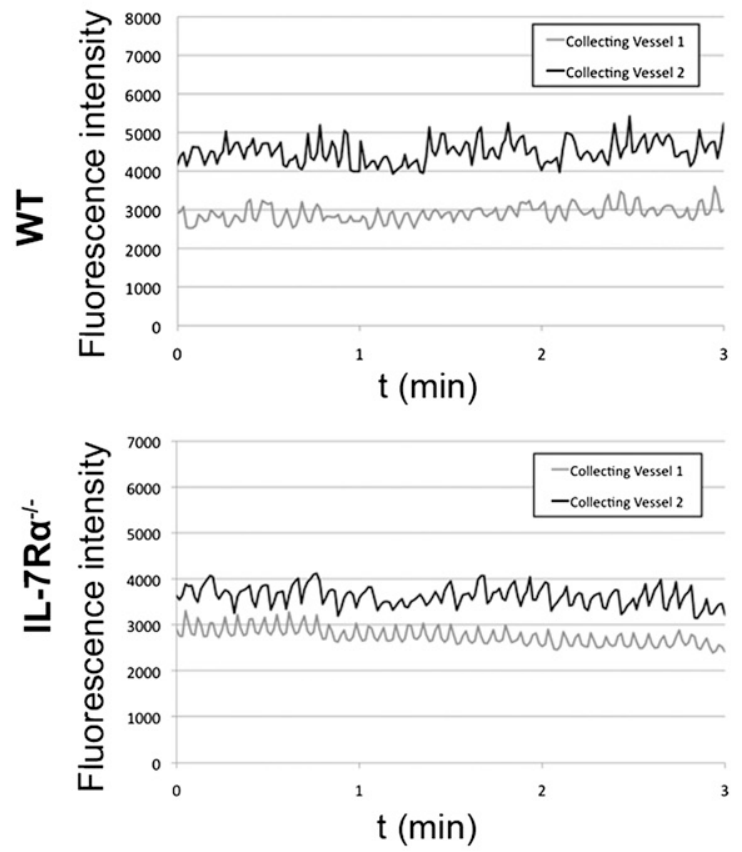

E

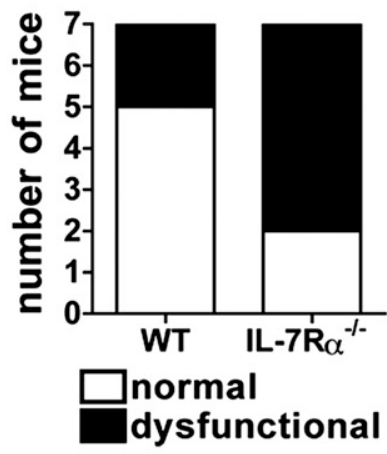

Figure 7. IL-7R $\alpha$ deficiency does not affect the pumping activity of lymphatic collectors but impairs uptake and transport in lymphatic initials. IL-7R ${ }^{-/-}$or WT mice $(n=7 / g e n o t y p e)$ were injected with a near-infrared dye (IRDye680) 20-kDa PEG conjugate into the hind paw and LVs in the upper and lower limb were examined under a stereomicroscope. (A) Representative images of the collecting LVs in the lower limb of an IL-7R $\alpha^{-1-}$ and a WT mouse. White circles indicate the region of the 2 collecting vessels in which pulse rates were analyzed. (B) Representative plots of the pulse rates measured in the 2 collecting vessels of a WT and an IL-7R $\alpha^{-/-}$mouse. (C) Quantification of the collecting LV contractility revealed no difference in pulse rates between IL-7R $\alpha^{-1-}$ and WT mice ( $\mathrm{n}=7$ mice/genotype). (D) Representative images of normal and dysfunctional lymph flow in the dorsal paw skin (upper limb) located proximal to the dye injection site. Under normal conditions (left), only deeper collecting vessels filled with dye are visible. By contrast, lymphatic dysfunction (right) is characterized by increased dye spreading through the initial lymphatic capillaries with evidence of leakage (white arrows). (E) Quantification of normal (white) and dysfunctional (black) lymphatic drainage in the dorsal paw skin proximal to the injection site ( $\mathrm{n}=7$ mice/ genotype). Scale bar represents $1 \mathrm{~mm}$.

Both transgenic overexpression of IL-7 and IL-7R $\alpha$ deficiency resulted in pronounced changes in the dermal lymphatic network in mice. In IL-7 $\mathrm{R}^{-1-}$ mice, the dermal LVs were organized in a highly branched, dense network of unusually thin LVs. Together with our in vitro data demonstrating a role for IL-7 in LEC tube formation and adhesion, the phenotype observed in IL-7R $\alpha^{-/-}$mice suggests that IL-7 signaling might contribute to lymphangiogenesis by stabilizing larger LVs. Notably, IL-7 had been previously linked with tumor lymphangiogenesis and thought to act indirectly by upregulating VEGF-D in IL-7R-expressing tumor cells. ${ }^{43,44}$ In light of our new in vivo findings, it is likely that IL-7 also displays direct lymphangiogenic activity on LECs in vivo, but we presently cannot exclude any indirect contribution from IL-7 responsive hematopoietic cells, such as $\mathrm{T}$ cells or LTi cells.

The most striking finding of our study was the observation that IL-7 supported LV drainage function. Whereas LV drainage was compromised in the skin of IL-7 $\mathrm{R}^{-/-}$mice, it was significantly enhanced in IL-7tg mice. Moreover, treatment of mice with IL-7/ anti-IL-7 complexes over a relatively short period of 7 days significantly enhanced lymphatic drainage. Intriguingly, drainage was enhanced at this time point, despite only subtle visible changes in the dermal LYVE- $1^{+}$lymphatic capillary network. It is therefore 
likely that the supportive effect of IL-7 on lymphatic drainage is not only a consequence of the morphologic, lymphangiogenesis-related changes in the LV network, but that IL-7 also stimulates lymphatic drainage in other ways. Our near-infrared imaging experiments clearly showed that the deficiency in lymphatic drainage observed in absence of IL-7R $\alpha$ signaling was not due to defects in the morphology or the pumping activity of lymphatic collecting vessels, but rather due to impaired lymph uptake or transport in lymphatic capillaries. The mechanism of lymph transport into initial lymphatic capillaries is not completely understood. ${ }^{45}$ Uptake is thought to mainly involve passive diffusion of fluids and macromolecules through the characteristic open flaps (named primary valves) present between neighboring capillary LECs. ${ }^{46}$ However, evidence for additional active, transcellular mechanisms has recently been reported. ${ }^{45}$ It is possible that IL-7 promotes drainage by increasing the integrity of the lymphatic vascular wall or by regulating the distinct organization of adhesion molecules and primary valves in lymphatic capillaries. ${ }^{46}$ Finally, the IL-7-induced upregulation of Prox-1 observed by us (supplemental Figure 3) and others ${ }^{29}$ might contribute to enhanced lymphatic drainage: analysis of mice heterozygous for Prox 1 deletion revealed that these mice had abnormalities in lymphatic flow and displayed increased vascular leakage. ${ }^{47}$

IL-7 is currently under clinical evaluation for its ability to enhance adaptive immunity in patients with viral infections or cancer. Therapeutic benefits of IL-7 treatment have mainly been attributed to druginduced improvement of T-cell survival, T-cell numbers, and T-cell repertoire diversity. ${ }^{12}$ Our data suggest that IL-7 therapy could have further beneficial, immune-activating effects by supporting lymphatic drainage function and thereby increasing the transport of antigen to draining LNs. On the other hand, our data also raise the concern that IL-7 therapy in the context of cancer might enhance tumor lymphangiogenesis and metastatic spread. Perhaps the most exciting translational aspect of our study is its potential implications for the treatment of LV dysfunction, namely lymphedema, for which only limited treatment options currently exist. ${ }^{48}$ In fact, the only factor with a broadly documented beneficial role in restoring $\mathrm{LV}$ function in animal models of lymphedema is VEGF-C. ${ }^{48,49}$ Given our new data on the pro-lymphangiogenic and drainage-promoting activity of IL-7, this cytokine might be an interesting new candidate to evaluate in future studies.

\section{Acknowledgments}

The authors thank Csaba Balazs (ETH Zurich) for developing the ImageJ-based script used for image analysis, Dr Anne Baerenwaldt (University of Basel) for precious help with mouse work, and Drs Paola Luciani and Jean-Christophe Leroux (ETH Zurich) for nearinfrared lymphatic tracers.

This work was supported by grants from the Swiss National Fund (310030_138330) (C.H.), ETH Zurich (0-20566-09) (C.H.) and the National Institutes of Health (EY017392) (L.C.).

\section{Authorship}

Contribution: M.I., D.A., and C.H. designed research, performed research, analyzed data, and wrote the paper; S.T.P., A.-H.W., S.H., T.E., L.C., G.B., and C.K. performed research and analyzed data; and D.F., L.O., B.L., L.S., and O.B. provided mice or essential reagents and discussed data.

Conflict-of-interest disclosure: The authors declare no competing financial interests.

Correspondence: Cornelia Halin, Institute of Pharmaceutical Sciences, ETH Zurich, Wolfgang-Pauli Str. 10, HCI H413, CH-8093 Zurich, Switzerland; e-mail: cornelia.halin@pharma.ethz.ch.

\section{References}

1. Schulte-Merker S, Sabine A, Petrova TV. Lymphatic vascular morphogenesis in development, physiology, and disease. J Cell Biol. 2011;193(4): 607-618.

2. Mäkinen T, Adams RH, Bailey J, et al. PDZ interaction site in ephrinB2 is required for the remodeling of lymphatic vasculature. Genes Dev. 2005;19(3):397-410.

3. Förster R, Braun A, Worbs T. Lymph node homing of $T$ cells and dendritic cells via afferent lymphatics. Trends Immunol. 2012;33(6): 271-280.

4. Johnson LA, Clasper S, Holt AP, Lalor PF, Baban D, Jackson DG. An inflammation-induced mechanism for leukocyte transmigration across lymphatic vessel endothelium. J Exp Med. 2006; 203(12):2763-2777.

5. Vigl B, Aebischer D, Nitschké M, et al. Tissue inflammation modulates gene expression of lymphatic endothelial cells and dendritic cell migration in a stimulus-dependent manner. Blood. 2011;118(1):205-215.

6. Furtado GC, Marinkovic T, Martin AP, et al. Lymphotoxin beta receptor signaling is required for inflammatory lymphangiogenesis in the thyroid. Proc Natl Acad Sci USA. 2007;104(12): 5026-5031.

7. Mounzer RH, Svendsen OS, Baluk $P$, et al. Lymphotoxin-alpha contributes to lymphangiogenesis. Blood. 2010;116(12): 2173-2182.
8. Chauhan SK, Jin Y, Goyal S, et al. A novel prolymphangiogenic function for Th17/IL-17. Blood. 2011;118(17):4630-4634.

9. Kataru RP, Kim H, Jang C, et al. T lymphocytes negatively regulate lymph node lymphatic vessel formation. Immunity. 2011;34(1):96-107.

10. Oka M, Iwata C, Suzuki HI, et al. Inhibition of endogenous TGF-beta signaling enhances lymphangiogenesis. Blood. 2008;111(9): 4571-4579.

11. Wagner LA, Brown T, Gil S, et al. The keratinocyte-derived cytokine IL-7 increases adhesion of the epidermal T cell subset to the skin basement membrane protein laminin-5. Eur $J$ Immunol. 1999;29(8):2530-2538.

12. Mackall CL, Fry TJ, Gress RE. Harnessing the biology of IL-7 for therapeutic application. Nat Rev Immunol. 2011;11(5):330-342.

13. Link A, Vogt TK, Favre $S$, et al. Fibroblastic reticular cells in lymph nodes regulate the homeostasis of naive T cells. Nat Immunol. 2007; 8(11):1255-1265

14. Malhotra D, Fletcher AL, Astarita J, et al; Immunological Genome Project Consortium. Transcriptional profiling of stroma from inflamed and resting lymph nodes defines immunological hallmarks. Nat Immunol. 2012;13(5):499-510.

15. Hara $T$, Shitara S, Imai K, et al. Identification of IL-7-producing cells in primary and secondary lymphoid organs using IL-7-GFP knock-in mice. J Immunol. 2012;189(4):1577-1584.
16. Onder L, Narang P, Scandella E, et al. IL-7producing stromal cells are critical for lymph node remodeling. Blood. 2012;120(24):4675-4683.

17. Surh CD, Sprent J. Homeostasis of naive and memory T cells. Immunity. 2008;29(6):848-862.

18. Schmutz S, Bosco N, Chappaz S, et al. Cutting edge: IL-7 regulates the peripheral pool of adult ROR gamma + lymphoid tissue inducer cells. J Immunol. 2009;183(4):2217-2221.

19. Vonarbourg C, Diefenbach A. Multifaceted roles of interleukin-7 signaling for the development and function of innate lymphoid cells. Semin Immunol. 2012;24(3):165-174.

20. Peschon JJ, Morrissey PJ, Grabstein $\mathrm{KH}$, et al. Early lymphocyte expansion is severely impaired in interleukin 7 receptor-deficient mice. J Exp Med. 1994;180(5):1955-1960.

21. von Freeden-Jeffry $U$, Vieira $P$, Lucian LA, McNeil $\mathrm{T}$, Burdach SE, Murray R. Lymphopenia in interleukin (IL)-7 gene-deleted mice identifies IL-7 as a nonredundant cytokine. J Exp Med. 1995; 181(4):1519-1526.

22. Hirakawa S, Hong YK, Harvey N, et al. Identification of vascular lineage-specific genes by transcriptional profiling of isolated blood vascular and lymphatic endothelial cells. $A m$ Pathol. 2003;162(2):575-586.

23. Meier D, Bornmann C, Chappaz S, et al. Ectopic lymphoid-organ development occurs through interleukin 7-mediated enhanced survival of lymphoid-tissue-inducer cells. Immunity. 2007; 26(5):643-654 
24. Repass JF, Laurent MN, Carter C, et al. IL7hCD25 and IL7-Cre BAC transgenic mouse lines: new tools for analysis of IL-7 expressing cells. Genesis. 2009;47(4):281-287.

25. Srinivas S, Watanabe T, Lin CS, et al. Cre reporter strains produced by targeted insertion of EYFP and ECFP into the ROSA26 locus. BMC Dev Biol. 2001;1:4.

26. Halin C, Detmar M. Chapter 1. Inflammation, angiogenesis, and lymphangiogenesis. Methods Enzymol. 2008;445:1-25.

27. Boyman O, Ramsey C, Kim DM, Sprent J, Surh CD. IL-7/anti-IL-7 mAb complexes restore T cell development and induce homeostatic $T$ cell expansion without lymphopenia. J Immunol. 2008; 180(11):7265-7275.

28. Jat PS, Noble MD, Ataliotis P, et al. Direct derivation of conditionally immortal cell lines from an $\mathrm{H}-2 \mathrm{~Kb}$-tsA58 transgenic mouse. Proc Nat Acad Sci USA. 1991;88(12):5096-5100.

29. Al-Rawi MA, Watkins G, Mansel RE, Jiang WG. The effects of interleukin-7 on the lymphangiogenic properties of human endothelial cells. Int J Oncol. 2005;27(3):721-730.

30. Avraamides CJ, Garmy-Susini B, Varner JA. Integrins in angiogenesis and lymphangiogenesis. Nat Rev Cancer. 2008;8(8):604-617.

31. Hong YK, Harvey N, Noh YH, et al. Prox1 is a master control gene in the program specifying lymphatic endothelial cell fate. Dev Dyn. 2002; 225(3):351-357.

32. Liao S, Ruddle NH. Synchrony of high endothelial venules and lymphatic vessels revealed by immunization. J Immunol. 2006;177(5): 3369-3379.

33. Huggenberger R, Siddiqui SS, Brander D, et al. An important role of lymphatic vessel activation in limiting acute inflammation. Blood. 2011;117(17): 4667-4678.

34. Mäkinen T, Jussila L, Veikkola T, et al. Inhibition of lymphangiogenesis with resulting lymphedema in transgenic mice expressing soluble VEGF receptor-3. Nat Med. 2001;7(2):199-205.

35. Avraham T, Yan A, Zampell JC, et al. Radiation therapy causes loss of dermal lymphatic vessels and interferes with lymphatic function by TGF. beta1-mediated tissue fibrosis. Am J Physiol Cell Physiol. 2010;299(3):C589-C605.

36. Miller CN, Hartigan-O'Connor DJ, Lee MS, et al. IL-7 production in murine lymphatic endothelial cells and induction in the setting of peripheral lymphopenia. Int Immunol. 2013;25(8):471-483.

37. Clement CC, Cannizzo ES, Nastke MD, et al. An expanded self-antigen peptidome is carried by the human lymph as compared to the plasma. PLOS ONE. 2010;5(3):e9863.

38. Fry TJ, Connick E, Falloon J, et al. A potential role for interleukin-7 in T-cell homeostasis. Blood. 2001;97(10):2983-2990.

39. Jacobs SR, Michalek RD, Rathmell JC. IL-7 is essential for homeostatic control of $\mathrm{T}$ cell metabolism in vivo. J Immunol. 2010;184(7): 3461-3469.

40. Liang B, Hara T, Wagatsuma K, et al. Role of hepatocyte-derived IL-7 in maintenance of intrahepatic NKT cells and T cells and development of B cells in fetal liver. J Immunol. 2012;189(9):4444-4450.

41. Bazigou E, Lyons OT, Smith A, et al. Genes regulating lymphangiogenesis control venous valve formation and maintenance in mice. $J$ Clin Invest. 2011;121(8):2984-2992.

42. Srinivasan RS, Oliver G. Prox1 dosage controls the number of lymphatic endothelial cell progenitors and the formation of the lymphovenous valves. Genes Dev. 2011;25(20): 2187-2197.

43. Al-Rawi MA, Watkins G, Mansel RE, Jiang WG. Interleukin 7 upregulates vascular endothelial growth factor $D$ in breast cancer cells and induces lymphangiogenesis in vivo. Br J Surg. 2005;92(3): 305-310.

44. Ming J, Zhang Q, Qiu X, Wang E. Interleukin 7/interleukin 7 receptor induce c-Fos/c-Jundependent vascular endothelial growth factor-D up-regulation: a mechanism of lymphangiogenesis in lung cancer. Eur $J$ Cancer. 2009;45(5):866-873.

45. Wiig $\mathrm{H}$, Swartz MA. Interstitial fluid and lymph formation and transport: physiological regulation and roles in inflammation and cancer. Physiol Rev. 2012;92(3):10051060.

46. Baluk $\mathrm{P}$, Fuxe J, Hashizume $\mathrm{H}$, et al. Functionally specialized junctions between endothelial cells of lymphatic vessels. J Exp Med. 2007;204(10): 2349-2362.

47. Harvey NL, Srinivasan RS, Dillard ME, et al. Lymphatic vascular defects promoted by Prox 1 haploinsufficiency cause adult-onset obesity. Nat Genet. 2005;37(10):1072-1081.

48. Tervala T, Suominen E, Saaristo A. Targeted treatment for lymphedema and lymphatic metastasis. Ann N Y Acad Sci. 2008;1131: 215-224.

49. Tammela T, Saaristo A, Holopainen T, et al. Therapeutic differentiation and maturation of lymphatic vessels after lymph node dissection and transplantation. Nat Med. 2007;13(12): 1458-1466. 